Article

\title{
Fourier Series for Singular Measures
}

\author{
John E. Herr ${ }^{1}$ and Eric S. Weber ${ }^{2, *}$ \\ 1 Department of Mathematics, Butler University, Indianapolis, IN 46208, USA; jeherr@butler.edu \\ 2 Department of Mathematics, Iowa State University, 396 Carver Hall, Ames, IA 50011, USA \\ * Correspondence: esweber@iastate.edu; Tel.: +1-515-294-8151
}

Academic Editors: Palle E.T. Jorgensen and Azita Mayeli

Received: 21 February 2017; Accepted: 24 March 2017; Published: 28 March 2017

\begin{abstract}
Using the Kaczmarz algorithm, we prove that for any singular Borel probability measure $\mu$ on $[0,1)$, every $f \in L^{2}(\mu)$ possesses a Fourier series of the form $f(x)=\sum_{n=0}^{\infty} c_{n} e^{2 \pi i n x}$. We show that the coefficients $c_{n}$ can be computed in terms of the quantities $\widehat{f}(n)=\int_{0}^{1} f(x) e^{-2 \pi i n x} d \mu(x)$. We also demonstrate a Shannon-type sampling theorem for functions that are in a sense $\mu$-bandlimited.
\end{abstract}

Keywords: Fourier series; singular measure; Kaczmarz algorithm

\section{Introduction}

For a Borel probability measure $\mu$, a spectrum is a sequence $\left\{\lambda_{n}\right\}_{n \in I}$ such that the functions $\left\{e^{2 \pi i \lambda_{n} x}: n \in I\right\} \subset L^{2}(\mu)$ constitute an orthonormal basis. If $\mu$ possesses a spectrum, we say $\mu$ is spectral, and then every $f \in L^{2}(\mu)$ possesses a (nonharmonic) Fourier series of the form $f(x)=\sum_{n \in I}\left\langle f(x), e^{2 \pi i \lambda_{n} x}\right\rangle e^{2 \pi i \lambda_{n} x}$.

In [1], Jorgensen and Pedersen considered the question of whether measures induced by iterated function systems on $\mathbb{R}^{d}$ are spectral. Remarkably, they demonstrated that the quaternary Cantor measure $\mu_{4}$ is spectral. Equally remarkably, they also showed that no three exponentials are orthogonal with respect to the ternary Cantor measure $\mu_{3}$, and hence $\mu_{3}$ is not spectral. The lack of a spectrum for $\mu_{3}$ motivated subsequent research to relax the orthogonality condition, instead searching for an exponential frame or Riesz basis, since an exponential frame would provide a Fourier series (see [2]) similar to the spectral case. Though these searches have yielded partial results, it is still an open question whether $L^{2}\left(\mu_{3}\right)$ possesses an exponential frame. It is known that there exist singular measures without exponential frames. In fact, Dutkay and Lai $[3,4]$ showed that self-affine measures induced by iterated function systems with no overlap cannot possess exponential frames if the probability weights are not equal.

In this paper, we demonstrate that the Kaczmarz algorithm educes another potentially fruitful substitute for exponential spectra and exponential frames: the "effective" sequences defined by Kwapień and Mycielski [5]. We show that the nonnegative integral exponentials in $L^{2}(\mu)$ for any singular Borel probability measure $\mu$ are such an effective sequence and that this effectivity allows us to define a Fourier series representation of any function in $L^{2}(\mu)$. This recovers a result of Poltoratskiı̌ [6] concerning the normalized Cauchy transform.

Definition 1. A sequence $\left\{f_{n}\right\}_{n=0}^{\infty}$ in a Hilbert space $\mathbb{H}$ is said to be Bessel if there exists a constant $B>0$ such that for any $x \in \mathbb{H}$,

$$
\sum_{n=0}^{\infty}\left|\left\langle x, f_{n}\right\rangle\right|^{2} \leq B\|x\|^{2} .
$$

This is equivalent to the existence of a constant $D>0$ such that 


$$
\left\|\sum_{n=0}^{K} c_{n} f_{n}\right\| \leq D \sqrt{\sum_{n=0}^{K}\left|c_{n}\right|^{2}}
$$

for any finite sequence $\left\{c_{0}, c_{1}, \ldots, c_{K}\right\}$ of complex numbers. The sequence is called a frame if in addition there exists a constant $A>0$ such that for any $x \in \mathbb{H}$,

$$
A\|x\|^{2} \leq \sum_{n=0}^{\infty}\left|\left\langle x, f_{n}\right\rangle\right|^{2} \leq B\|x\|^{2} .
$$

If $A=B$, then the frame is said to be tight. If $A=B=1$, then $\left\{f_{n}\right\}_{n=0}^{\infty}$ is a Parseval frame. The constant $A$ is called the lower frame bound and the constant $B$ is called the upper frame bound or Bessel bound.

Definition 2. The Fourier-Stieltjes transform of a finite Borel measure $\mu$ on $[0,1)$, denoted $\widehat{\mu}$, is defined by

$$
\widehat{\mu}(x):=\int_{0}^{1} e^{-2 \pi i x y} d \mu(y) .
$$

\subsection{Effective Sequences}

Let $\left\{\varphi_{n}\right\}_{n=0}^{\infty}$ be a linearly dense sequence of unit vectors in a Hilbert space $\mathbb{H}$. Given any element $x \in \mathbb{H}$, we may define a sequence $\left\{x_{n}\right\}_{n=0}^{\infty}$ in the following manner:

$$
\begin{aligned}
x_{0}, & =\left\langle x, \varphi_{0}\right\rangle \varphi_{0} \\
x_{n} & =x_{n-1}+\left\langle x-x_{n-1}, \varphi_{n}\right\rangle \varphi_{n} .
\end{aligned}
$$

If $\lim _{n \rightarrow \infty}\left\|x-x_{n}\right\|=0$ regardless of the choice of $x$, then the sequence $\left\{\varphi_{n}\right\}_{n=0}^{\infty}$ is said to be effective.

The above formula is known as the Kaczmarz algorithm. In 1937, Stefan Kaczmarz [7] proved the effectivity of linearly dense periodic sequences in the finite-dimensional case. In 2001, these results were extended to infinite-dimensional Banach spaces under certain conditions by Kwapień and Mycielski [5] (see also [8]). These two also gave the following formula for the sequence $\left\{x_{n}\right\}_{n=0}^{\infty}$, which we state here for the Hilbert space setting: define

$$
\begin{aligned}
& g_{0}=\varphi_{0} \\
& g_{n}=\varphi_{n}-\sum_{i=0}^{n-1}\left\langle\varphi_{n}, \varphi_{i}\right\rangle g_{i} .
\end{aligned}
$$

Then,

$$
x_{n}=\sum_{i=0}^{n}\left\langle x, g_{i}\right\rangle \varphi_{i}
$$

As shown by [5], and also more clearly for the Hilbert space setting by [9], we have

$$
\|x\|^{2}-\lim _{n \rightarrow \infty}\left\|x-x_{n}\right\|^{2}=\sum_{n=0}^{\infty}\left|\left\langle x, g_{n}\right\rangle\right|^{2},
$$

from which it follows that $\left\{\varphi_{n}\right\}_{n=0}^{\infty}$ is effective if and only if

$$
\sum_{n=0}^{\infty}\left|\left\langle x, g_{n}\right\rangle\right|^{2}=\|x\|^{2}
$$

That is to say, $\left\{\varphi_{n}\right\}_{n=0}^{\infty}$ is effective if and only if the associated sequence $\left\{g_{n}\right\}_{n=0}^{\infty}$ is a Parseval frame. 
If $\left\{\varphi_{n}\right\}_{n=0}^{\infty}$ is effective, then Label (4) implies that for any $x \in \mathbb{H}, \sum_{i=0}^{\infty}\left\langle x, g_{i}\right\rangle \varphi_{i}$ converges to $x$ in norm, and, as noted, $\left\{g_{n}\right\}_{n=0}^{\infty}$ is a Parseval frame. This does not mean that $\left\{g_{n}\right\}_{n=0}^{\infty}$ and $\left\{\varphi_{n}\right\}_{n=0}^{\infty}$ are dual frames, since $\left\{\varphi_{n}\right\}_{n=0}^{\infty}$ need not even be a frame. However, $\left\{\varphi_{n}\right\}_{n=0}^{\infty}$ and $\left\{g_{n}\right\}_{n=0}^{\infty}$ are pseudo-dual in the following sense, first given by Li and Ogawa in [10]:

Definition 3. Let $\mathcal{H}$ be a separable Hilbert space. Two sequences $\left\{\varphi_{n}\right\}$ and $\left\{\varphi_{n}^{\star}\right\}$ in $\mathcal{H}$ form a pair of pseudoframes for $\mathcal{H}$ if for all $x, y \in \mathcal{H},\langle x, y\rangle=\sum_{n}\left\langle x, \varphi_{n}^{\star}\right\rangle\left\langle\varphi_{n}, y\right\rangle$.

All frames are pseudoframes, but not the converse. Observe that if $x, y \in \mathbb{H}$ and $\left\{\varphi_{n}\right\}_{n=0}^{\infty}$ is effective, then

$$
\begin{aligned}
\langle x, y\rangle & =\left\langle\sum_{m=0}^{\infty}\left\langle x, g_{m}\right\rangle \varphi_{m}, y\right\rangle \\
& =\sum_{m=0}^{\infty}\left\langle x, g_{m}\right\rangle\left\langle\varphi_{m}, y\right\rangle,
\end{aligned}
$$

and so $\left\{\varphi_{n}\right\}_{n=0}^{\infty}$ and $\left\{g_{n}\right\}_{n=0}^{\infty}$ are pseudo-dual.

Of course, since $\left\{g_{n}\right\}_{n=0}^{\infty}$ is a Parseval frame, it is a true dual frame for itself.

\section{Main Results}

From this point forward, we shall use the notation $e_{\lambda}(x):=e^{2 \pi i \lambda x}$. Our main result is as follows.

Theorem 1. If $\mu$ is a singular Borel probability measure on $[0,1)$, then the sequence $\left\{e_{n}\right\}_{n=0}^{\infty}$ is effective in $L^{2}(\mu)$. As a consequence, any element $f \in L^{2}(\mu)$ possesses a Fourier series

$$
f(x)=\sum_{n=0}^{\infty} c_{n} e^{2 \pi i n x}
$$

where

$$
c_{n}=\int_{0}^{1} f(x) \overline{g_{n}(x)} d \mu(x)
$$

, and $\left\{g_{n}\right\}_{n=0}^{\infty}$ is the sequence associated to $\left\{e_{n}\right\}_{n=0}^{\infty}$ via Equation (3). The sum converges in norm, and Parseval's identity $\|f\|^{2}=\sum_{n=0}^{\infty}\left|c_{n}\right|^{2}$ holds.

Our proof proceeds in a series of lemmas. First, in order to show completeness of $\left\{e_{n}\right\}_{n=0}^{\infty}$, we appeal to the well-known theorem of Frigyes and Marcel Riesz [11]:

Theorem 2. [F. and M. Riesz] Let $\mu$ be a complex Borel measure on $[0,1)$. If

$$
\int_{0}^{1} e^{2 \pi i n x} d \mu(x)=0
$$

for all $n \in \mathbb{N}$, then $\mu$ is absolutely continuous with respect to Lebesgue measure.

From this theorem, we prove the desired lemma:

Lemma 1. If $\mu$ is a singular Borel measure on $[0,1)$, then $\left\{e_{n}\right\}_{n=0}^{\infty}$ is linearly dense in $L^{2}(\mu)$. 
Proof. Assume, for the sake of contradiction, that $\overline{\operatorname{span}}\left(\left\{e_{n}\right\}_{n=0}^{\infty}\right) \neq L^{2}(\mu)$. Then, there exists some $f \in L^{2}(\mu)$ such that $f \in \overline{\operatorname{span}}\left(\left\{e_{n}\right\}_{n=0}^{\infty}\right)^{\perp}$. Then, for any $n \in \mathbb{N}$, we have

$$
\int_{0}^{1} e^{2 \pi i n x} \overline{f(x)} d \mu(x)=0
$$

By the F. and M. Riesz Theorem, this implies that $\bar{f} d \mu$ is absolutely continuous with respect to Lebesgue measure $d \lambda$. Since $\bar{f} d \mu<<d \lambda$ and $\bar{f} d \mu \perp d \lambda$, it follows by uniqueness in Lebesgue's Decomposition Theorem that $\bar{f} d \mu \equiv 0$. Thus, $f=0$ almost everywhere with respect to $\mu$, which is a contradiction. Therefore, $\overline{\operatorname{span}}\left(\left\{e_{n}\right\}_{n=0}^{\infty}\right)=L^{2}(\mu)$.

Definition 4 (Stationary Sequences). A sequence $\left\{\varphi_{k}\right\}_{k=0}^{\infty}$ in a Hilbert space is said to be stationary if $\left\langle\varphi_{k+m}, \varphi_{l+m}\right\rangle=\left\langle\varphi_{k}, \varphi_{l}\right\rangle$ for any nonnegative integers $k, l$, and $m$.

As noted in [5], given a stationary sequence $\left\{\varphi_{n}\right\}_{n=0}^{\infty}$ and $a_{m}$ defined by $a_{m}:=\left\langle\varphi_{k}, \varphi_{k+m}\right\rangle$, where $k$ is any nonnegative integer $k \geq-m$, Bochner's Theorem implies the existence of a unique positive measure $\sigma$ on $\mathbb{T}$ such that

$$
a_{m}=\int_{\mathbb{T}} \bar{z}^{m} \sigma(d z)=\int_{0}^{1} e^{-2 \pi i m x} d \sigma(x) \quad \text { for each } m \in \mathbb{Z} .
$$

This measure $\sigma$ is called the spectral measure of the stationary sequence $\left\{\varphi_{n}\right\}$.

We shall make use of the following theorem from [5]:

Theorem 3 (Kwapień and Mycielski). A stationary sequence of unit vectors that is linearly dense in a Hilbert space is effective if and only if its spectral measure either coincides with the normalized Lebesgue measure or is singular with respect to Lebesgue measure.

It can be shown that if $\mu$ is a Borel probability measure on $[0,1)$, then the function $G(z)=1-\left(\int_{0}^{1} \frac{1}{1-z e^{-2 \pi i x}} d \mu(x)\right)^{-1}$ is holomorphic on $\mathbb{D}$ with $|G(z)| \leq 1$ and also satisfies

$$
\operatorname{Re}\left(\frac{1+G(z)}{1-G(z)}\right)=\int_{0}^{1} \frac{1-|z|^{2}}{\left|e^{2 \pi i x}-z\right|^{2}} d \mu(x),
$$

where the function on the left the unique nonnegative harmonic function on $\mathbb{D}$ associated to $\mu$ by the Herglotz representation theorem [12]. Suppose $\left\{\varphi_{k}\right\}$ is a stationary sequence of linearly-dense unit vectors, $\mu=\sigma$ is the spectral measure of $\left\{\varphi_{k}\right\}$, and $G(z)=\sum c_{n} z^{n}$ is constructed as above. The proof of the Kwapień-Mycielski Theorem works by using some intricate algebra to show that either $\sigma$ is Lebesgue measure, or that by applying the Kaczmarz algorithm based on $\left\{\varphi_{k}\right\}$ to any one of the $\varphi_{k}$ themselves Label (4), one obtains:

$$
\left\|\varphi_{k}-\sum_{i=0}^{n}\left\langle\varphi_{k}, g_{i}\right\rangle \varphi_{i}\right\|^{2} \lesssim 1-\sum_{j=1}^{n}\left|c_{j}\right|^{2} .
$$

This makes effectivity of $\left\{\varphi_{k}\right\}$ equivalent to $\sum_{j=1}^{\infty}\left|c_{j}\right|^{2}=1$. Since $G(z)$ is already bounded by 1 , the sum is 1 if and only if $G(z)$ is inner, which, in turn by the Herglotz representation combined with Fatou's Theorem, is equivalent to $\sigma$ being singular [12]. See [13] for a complete proof.

We are now ready to prove Theorem 1 . 
Proof of Theorem 1. By Lemma 1, the sequence $\left\{e_{n}\right\}_{n=0}^{\infty}$ is linearly dense in $L^{2}(\mu)$. It consists of unit vectors because $\mu$ is a probability measure. We see that, for all $k, l, m \in \mathbb{N}_{0}$,

$$
\left\langle e_{k+m}, e_{l+m}\right\rangle=\int_{[0,1)} e^{2 \pi i(k-l) x} d \mu(x)=\left\langle e_{k}, e_{l}\right\rangle
$$

Thus, $\left\{e_{n}\right\}_{n=0}^{\infty}$ is stationary in $L^{2}(\mu)$, and, moreover, $\mu$ is its spectral measure. It then follows from the theorem of Kwapien and Mycielski that $\left\{e_{n}\right\}_{n=0}^{\infty}$ is effective in $L^{2}(\mu)$.

Since $\left\{e_{n}\right\}_{n=0}^{\infty}$ is effective, given any $f \in L^{2}(\mu)$, we have that the Kaczmarz algorithm sequence defined recursively by

$$
\begin{aligned}
f_{0}, & =\left\langle f, e_{0}\right\rangle e_{0}, \\
f_{n} & =f_{n-1}+\left\langle f-f_{n-1}, e_{n}\right\rangle e_{n}
\end{aligned}
$$

satisfies

$$
\lim _{n \rightarrow \infty}\left\|f-f_{n}\right\|=0
$$

We recall that

$$
f_{n}=\sum_{i=0}^{n}\left\langle f, g_{i}\right\rangle e_{i}
$$

where the sequence $\left\{g_{n}\right\}_{n=0}^{\infty}$ is the sequence associated to the sequence $\left\{e_{n}\right\}_{n=0}^{\infty}$ by Label (3). Hence,

$$
f=\sum_{i=0}^{\infty}\left\langle f, g_{i}\right\rangle e_{i}
$$

Setting $c_{n}=\left\langle f, g_{n}\right\rangle=\int_{0}^{1} f(x) \overline{g_{n}(x)} d \mu(x)$ yields

$$
f(x)=\sum_{n=0}^{\infty} c_{n} e^{2 \pi i n x}
$$

where the convergence is in the norm. Furthermore, since $\left\{e_{n}\right\}_{n=0}^{\infty}$ is effective, by Label (5), $\left\{g_{n}\right\}_{n=0}^{\infty}$ is a Parseval frame. Thus,

$$
\sum_{n=0}^{\infty}\left|c_{n}\right|^{2}=\sum_{n=0}^{\infty}\left|\left\langle f, g_{n}\right\rangle\right|^{2}=\|f\|^{2}
$$

This completes the proof.

Since the ternary Cantor measure $\mu_{3}$ is a singular probability measure, Theorem 1 demonstrates that any $f \in L^{2}\left(\mu_{3}\right)$ possesses a Fourier series of the form prescribed by the theorem. This comes despite the fact that $\mu_{3}$ does not possess an orthogonal basis of exponentials. It is still unknown whether $L^{2}\left(\mu_{3}\right)$ even possesses an exponential frame.

The sequence $\left\{e_{n}\right\}_{n=0}^{\infty}$ of exponentials is effective in $L^{2}(\mu)$ for all singular Borel probability measures $\mu$, but it is Bessel in none of them. Indeed, if it were Bessel, $\mu$ would be absolutely continuous rather than singular by Theorem 3.10 of [14]. In fact, Proposition 3.10 in [15] demonstrates an example of a (singular) measure $\mu$ and a function $f \in L^{2}(\mu)$ where $\sum_{n=0}^{\infty}|\widehat{f}(n)|^{2}=+\infty$. Therefore, it is not possible for $\left\{e_{n}\right\}_{n=0}^{\infty}$ to be a frame in $L^{2}(\mu)$. However, by a remark in [10], since $\left\{e_{n}\right\}_{n=0}^{\infty}$ is pseudo-dual to the (in this case Parseval) frame $\left\{g_{n}\right\}_{n=0}^{\infty}$, the upper frame bound for $\left\{g_{n}\right\}_{n=0}^{\infty}$ implies a lower frame bound for $\left\{e_{n}\right\}_{n=0}^{\infty}$.

Moreover, some of the examples in [3,4] of measures that do not possess an exponential frame are singular, and hence if we normalize them to be probability measures, Theorem 1 applies.

We shall give a somewhat more explicit formula for the coefficients $c_{n}$. We will require a lemma to do this, but first we discuss some notation: 
Remark 1. Recall that a composition of a positive integer $n$ is an ordered arrangement of positive integers that sum to $n$. Whereas for a partition the order in which the terms appear does not matter, two sequences having the same terms but in a different order constitute different compositions. We will think of compositions of $n$ as tuples of positive integers whose entries sum to $n$. The set of compositions of $n$ will be denoted $P_{n}$. In other words,

$$
P_{n}:=\left\{\left(p_{1}, p_{2}, \ldots, p_{k}\right) \mid k \in \mathbb{N},\left(p_{1}, p_{2}, \ldots, p_{k}\right) \in \mathbb{N}^{k}, p_{1}+p_{2}+\cdots+p_{k}=n\right\} .
$$

Thus, we have $P_{1}=\{(1)\}, P_{2}=\{(2),(1,1)\}, P_{3}=\{(3),(1,2),(2,1),(1,1,1)\}$, etc. The length of $a$ tuple $p \in P_{n}$ will be denoted $l(p)$, i.e. $p=\left(p_{1}, p_{2}, \ldots, p_{l(p)}\right) \in \mathbb{N}^{l(p)}$.

Lemma 2. Let $\mu$ be a Borel probability measure on $[0,1)$ with Fourier-Stieltjes transform $\widehat{\mu}$. Define $\alpha_{0}=1$, and for $n \geq 1$, let

$$
\alpha_{n}=\sum_{p \in P_{n}}(-1)^{l(p)} \prod_{j=1}^{l(p)} \widehat{\mu}\left(p_{j}\right) .
$$

Let $\left\{g_{n}\right\}_{n=0}^{\infty}$ be as defined in Label (3). Then, for all $n \in \mathbb{N}_{0}$,

$$
g_{n}=\sum_{j=0}^{n} \overline{\alpha_{n-j}} e_{j}
$$

Proof. Clearly, $g_{0}=e_{0}$ and $g_{1}=e_{1}-\left\langle e_{1}, e_{0}\right\rangle e_{0}=e_{1}-\overline{\widehat{\mu}(1)} e_{0}$. We have that $P_{1}=\{(1)\}$, so

$$
\alpha_{1}=(-1)^{1} \widehat{\mu}(1)=-\widehat{\mu}(1) .
$$

Therefore, the conclusion holds for $n=0,1$. Suppose that the conclusion holds up to some $n \in \mathbb{N}$. We have that

$$
\begin{aligned}
g_{n+1} & =e_{n+1}-\sum_{j=0}^{n}\left\langle e_{n+1}, e_{j}\right\rangle g_{j} \\
& =e_{n+1}-\sum_{j=0}^{n} \overline{\widehat{\mu}(n+1-j)} g_{j} \\
& =e_{n+1}-\sum_{j=0}^{n} \overline{\widehat{\mu}(n+1-j)}\left(\sum_{k=0}^{j} \overline{\alpha_{j-k}} e_{k}\right) \\
& =e_{n+1}-\sum_{j=0}^{n} \sum_{k=0}^{j} \overline{\widehat{\mu}(n+1-j) \overline{\alpha_{j-k}} e_{k}} \\
& =e_{n+1}-\sum_{k=0}^{n} \sum_{j=k}^{n} \overline{\widehat{\mu}(n+1-j)} \overline{\alpha_{j-k}} e_{k} .
\end{aligned}
$$

Thus, it remains only to show that

$$
\alpha_{n+1-k}=-\sum_{j=k}^{n} \widehat{\mu}(n+1-j) \alpha_{j-k} .
$$


We have:

$$
\begin{aligned}
-\sum_{j=k}^{n} \widehat{\mu}(n+1-j) \alpha_{j-k} & =-\sum_{j=k}^{n} \widehat{\mu}(n+1-j) \sum_{p \in P_{j-k}}(-1)^{l(p)} \prod_{w=1}^{l(p)} \widehat{\mu}\left(p_{w}\right) \\
& =\sum_{j=k}^{n} \sum_{p \in P_{j-k}}(-1)^{l(p)+1} \widehat{\mu}(n+1-j) \prod_{w=1}^{l(p)} \widehat{\mu}\left(p_{w}\right) \\
& =\sum_{j=1}^{n+1-k} \sum_{p \in P_{n-k+1-j}}(-1)^{l(p)+1} \widehat{\mu}(j) \prod_{w=1}^{l(p)} \widehat{\mu}\left(p_{w}\right) .
\end{aligned}
$$

The last equality is obtained by reindexing the sum $j \mapsto n+1-j$. Now, if $p=\left(p_{1}, \ldots, p_{l(p)}\right) \in P_{n}$, then it is obvious that $p_{1} \in\{1,2, \ldots, n\}$ and $\left(p_{2}, p_{3}, \ldots, p_{l(p)}\right) \in P_{n-p_{1}}$ (where we define $\left.P_{0}=\varnothing\right)$. Conversely, if $p_{1} \in\{1,2, \ldots, n\}$ and $\left(p_{2}, p_{3}, \ldots, p_{l(p)}\right) \in P_{n-p_{1}}$, then clearly $\left(p_{1}, p_{2}, \ldots, p_{l(p)}\right) \in P_{n}$. Thus, it follows that

$$
-\sum_{j=k}^{n} \widehat{\mu}(n+1-j) \alpha_{j-k}=\sum_{p \in P_{n+1-k}}(-1)^{l(p)} \prod_{w=1}^{l(p)} \widehat{\mu}\left(p_{2}\right)=\alpha_{n+1-k} .
$$

This completes the proof.

Remark 2. Lemma 2 can easily be generalized to any Hilbert space setting in which the $\left\{g_{n}\right\}_{n=0}^{\infty}$ are induced by a stationary sequence $\left\{\varphi_{n}\right\}_{n=0}^{\infty}$ simply by replacing $\widehat{\mu}(m)$ with $a_{m}$ in all instances, where the $a_{m}$ are as defined after Definition 4.

It should be pointed out that sequence of scalars $\left\{\alpha_{n}\right\}_{n=0}^{\infty}$ depends only on the measure $\mu$. In a general Hilbert space setting where we may not have stationarity, an expansion of the $\left\{g_{n}\right\}$ in terms of the sequence $\left\{\varphi_{n}\right\}$ to which they are associated by Label (2) can be described by using inversion of an infinite lower-triangular Gram matrix. For a treatment, see [9].

Definition 5. Define a Fourier transform of $f$ by

$$
\mathcal{F} f(y)=\widehat{f}(y):=\int_{0}^{1} f(x) e^{-2 \pi i y x} d \mu(x)
$$

Observe that

$$
|\mathcal{F} f(y)|=\left|\left\langle f, e_{y}\right\rangle\right| \leq\|f\|_{L^{2}(\mu)} \cdot\left\|e_{y}\right\|_{L^{2}(\mu)}=\|f\|_{L^{2}(\mu)} \cdot
$$

Thus, $\mathcal{F}$ is a linear operator from $L^{2}(\mu)$ to $L^{\infty}(\mathbb{R})$ with operator norm $\|\mathcal{F}\|=1$.

Corollary 1. Assume the conditions and definitions of Theorem 1. Then, the coefficients $c_{n}$ may be expressed

$$
c_{n}=\sum_{j=0}^{n} \alpha_{n-j} \widehat{f}(j),
$$

and as a result

$$
f(x)=\sum_{n=0}^{\infty}\left(\sum_{j=0}^{n} \alpha_{n-j} \widehat{f}(j)\right) e^{2 \pi i n x}
$$

where the convergence is in norm. 
Proof. We compute:

$$
c_{n}=\left\langle f, g_{n}\right\rangle=\left\langle f, \sum_{j=0}^{n} \overline{\alpha_{n-j}} e_{j}\right\rangle=\sum_{j=0}^{n} \alpha_{n-j} \widehat{f}(j) .
$$

The second formula then follows by substitution into Label (6).

\subsection{Non-Uniqueness of Fourier Coefficients}

We begin with an example. In [1], it was shown that the quaternary Cantor measure $\mu_{4}$ possesses an orthonormal basis of exponentials. This basis is $\left\{e_{\lambda}\right\}_{\lambda \in \Lambda}$, where the spectrum $\Lambda$ is given by

$$
\Lambda=\left\{\sum_{n=0}^{k} \alpha_{n} 4^{n}: \alpha_{n} \in\{0,1\}, k \in \mathbb{N}_{0}\right\}=\{0,1,4,5,16,17,20,21, \ldots\} .
$$

As a result, any vector $f \in L^{2}\left(\mu_{4}\right)$ may be written as

$$
f=\sum_{\lambda \in \Lambda}\left\langle f, e_{\lambda}\right\rangle e_{\lambda}
$$

where the convergence is in the $L^{2}\left(\mu_{4}\right)$ norm. Notice that if we define a sequence of vectors $\left\{h_{n}\right\}_{n=0}^{\infty}$ by

$$
h_{n}= \begin{cases}e_{n}, & \text { if } n \in \Lambda, \\ 0, & \text { otherwise }\end{cases}
$$

we have that

$$
\sum_{n=0}^{\infty}\left\langle f, h_{n}\right\rangle e_{n}=\sum_{\lambda \in \Lambda}\left\langle f, e_{\lambda}\right\rangle e_{\lambda}=f .
$$

On the other hand, since $\mu_{4}$ is a singular probability measure, by Theorem 1 , we also have

$$
f=\sum_{n=0}^{\infty} c_{n} e_{n}=\sum_{n=0}^{\infty}\left\langle f, g_{n}\right\rangle e_{n}
$$

It can easily be checked that $h_{0}=g_{0}=e_{0}$ and $h_{1}=g_{1}=e_{1}$, but that $g_{2} \neq h_{2}=0$. Thus, the sequences $\left\{g_{n}\right\}$ and $\left\{h_{n}\right\}$ yield different expansions for general $f \in L^{2}\left(\mu_{4}\right)$.

We can again use the Kaczmarz algorithm to generate a large class of sequences $\left\{h_{n}\right\}$ such that $\Sigma\left\langle f, h_{n}\right\rangle e_{n}=f$ in the $L^{2}(\mu)$ norm as follows. We use $\langle\cdot, \cdot\rangle_{\mu}$ to denote the scalar product in $L^{2}(\mu)$.

Theorem 4. Let $\mu$ be a singular Borel probability measure on $[0,1)$. Let $v$ be another singular Borel probability measure on $[0,1)$ such that $v \perp \mu$. Let $0<\eta \leq 1$, and define $\lambda:=\eta \mu+(1-\eta) v$. Let $\left\{h_{n}\right\}$ be the sequence associated to $\left\{e_{n}\right\}$ in $L^{2}(\lambda)$ via the Kaczmarz algorithm in Equation (3). Then, for all $f \in L^{2}(\mu)$,

$$
f=\sum_{n=0}^{\infty}\left\langle f, \eta h_{n}\right\rangle_{\mu} e_{n}
$$

in the $L^{2}(\mu)$ norm. Moreover, if $\lambda^{\prime}=\eta^{\prime} \mu+\left(1-\eta^{\prime}\right) v^{\prime}$ also satisfies the hypotheses, then $\lambda^{\prime} \neq \lambda$ implies $\left\{\eta^{\prime} h_{n}^{\prime}\right\} \neq\left\{\eta h_{n}\right\}$ in $L^{2}(\mu)$.

Proof. Since $\lambda$ is a singular Borel probability measure, the exponentials $\left\{e_{n}\right\}_{n=0}^{\infty}$ are effective in $L^{2}(\lambda)$. Let $\left\{h_{n}\right\}$ denote the sequence associated to $\left\{e_{n}\right\}$ in $L^{2}(\lambda)$ via Equation (3). Let $f \in L^{2}(\mu)$, and define $\tilde{f}=f \cdot \chi_{\operatorname{supp}(\mu)}$. Clearly, $\tilde{f} \in L^{2}(\lambda)$. 
We have that

$$
\tilde{f}=\sum_{n=0}^{\infty}\left\langle\tilde{f}, h_{n}\right\rangle_{\lambda} e_{n}
$$

in the $L^{2}(\lambda)$ norm. Now, note that

$$
\begin{aligned}
\left\langle f, \eta h_{n}\right\rangle_{\mu} & =\int_{0}^{1} f(x) \overline{\eta h_{n}(x)} d \mu(x) \\
& =\int_{\operatorname{supp}(\mu)} f(x) \overline{h_{n}(x)} d \lambda \\
& =\left\langle\tilde{f}, h_{n}\right\rangle_{\lambda} .
\end{aligned}
$$

Therefore,

$$
\lim _{N \rightarrow \infty}\left\|\tilde{f}-\sum_{n=0}^{N}\left\langle f, \eta h_{n}\right\rangle_{\mu} e_{n}\right\|_{L^{2}(\lambda)}^{2}=0
$$

Since

$$
\left\|f-\sum_{n=0}^{N}\left\langle f, \eta h_{n}\right\rangle_{\mu} e_{n}\right\|_{L^{2}(\mu)}^{2} \leq \frac{1}{\eta}\left\|\tilde{f}-\sum_{n=0}^{N}\left\langle f, \eta h_{n}\right\rangle_{\mu} e_{n}\right\|_{L^{2}(\lambda)}^{2}
$$

Equation (8) follows with convergence in $L^{2}(\mu)$.

It remains only to show that different measures $\lambda$ generate different sequences $\left\{\eta h_{n}\right\}$. Therefore, suppose $v^{\prime}$ is another singular Borel probability measure on $[0,1)$ such that $v^{\prime} \perp \mu$, and let $0<\eta^{\prime} \leq 1$. Set $\lambda^{\prime}=\eta^{\prime} \mu+\left(1-\eta^{\prime}\right) v^{\prime}$, and let $\left\{h_{n}^{\prime}\right\}$ be the sequence associated to $\left\{e_{n}\right\}$ in $L^{2}\left(\lambda^{\prime}\right)$ via Equation (3). Suppose that $\lambda \neq \lambda^{\prime}$. We wish to show that $\left\{\eta h_{n}\right\} \neq\left\{\eta^{\prime} h_{n}^{\prime}\right\}$ in $L^{2}(\mu)$.

If $\eta \neq \eta^{\prime}$, then $\eta h_{0}=\eta e_{0} \neq \eta^{\prime} e_{0}=\eta^{\prime} h_{0}^{\prime}$ in $L^{2}(\mu)$. Therefore, assume that $\eta=\eta^{\prime}$. By virtue of the F. and M. Riesz Theorem, since $\lambda \neq \lambda^{\prime}$, there must exist an integer $n$ such that $\widehat{\lambda}(n) \neq \widehat{\lambda}^{\prime}(n)$. Following [9], we define a lower-triangular Gram matrix $G$ of the nonnegative integral exponentials by

$$
(G)_{i j}= \begin{cases}\left\langle e_{i}, e_{j}\right\rangle=\hat{\lambda}(j-i), & \text { if } i \geq j \\ 0, & \text { otherwise }\end{cases}
$$

and then the inverse of this matrix determines the sequence $\left\{h_{n}\right\}$ associated to $\left\{e_{n}\right\}$ in $L^{2}(\lambda)$ via $h_{n}=\sum_{i=0}^{n} \overline{\alpha_{n-i}} e_{i}$, where $\alpha_{n-i}=\overline{\left(G^{-1}\right)_{n i}}$. See [9] for details. ( $G$ and $G^{-1}$ are stratified since $\left\{e_{n}\right\}$ is stationary.) Therefore, the sequences of scalars $\left\{\alpha_{n}\right\}_{n=0}^{\infty}$ and $\left\{\alpha_{n}^{\prime}\right\}_{n=0}^{\infty}$ induced by $\lambda$ and $\lambda^{\prime}$, respectively, in Lemma 2 differ. Let $n$ be the smallest positive integer such that $\alpha_{n} \neq \alpha_{n}^{\prime}$. Then, since $\eta=\eta^{\prime}$, we have

$$
\eta^{\prime} h_{n}^{\prime}-\eta h_{n}=\eta \sum_{j=0}^{n}\left(\overline{\alpha_{n-j}^{\prime}}-\overline{\alpha_{n-j}}\right) e_{j}=\eta\left(\overline{\alpha_{n}-\alpha_{n}^{\prime}}\right) e_{0} \neq 0 .
$$

Thus, $\left\{\eta h_{n}\right\}$ and $\left\{\eta^{\prime} h_{n}^{\prime}\right\}$ are distinct sequences in $L^{2}(\mu)$.

Remark 3. We note that any convex combination of sequences $\left\{h_{n}\right\}$ that satisfy Equation (8) will again satisfy that equation.

In general, for a fixed $f \in L^{2}(\mu)$, the set of coefficient sequences $\left\{d_{n}\right\}$ that satisfy $f=\sum_{n=0}^{\infty} d_{n} e_{n}$ can be parametrized by sequences $\left\{\gamma_{n}\right\}$ of scalars satisfying $\sum_{n=0}^{\infty} \gamma_{n} e_{n}=0$ via $d_{n}=\left\langle f, g_{n}\right\rangle_{\mu}+\gamma_{n}$. Clearly, Theorem 4 is not a complete description of all Fourier series expansions for $f$.

\subsection{Connection to the Normalized Cauchy Transform}

The series $\sum_{n=0}^{\infty}\left\langle f, g_{n}\right\rangle e_{n}$ given by Theorem 1 is the boundary function of the analytic function $\sum_{n=0}^{\infty}\left\langle f, g_{n}\right\rangle z^{n}$ on $\mathbb{D}$. This function is in the classical $H^{2}$ Hardy space since the coefficients are square 
summable. An intriguing connection between the Kaczmarz algorithm and de Branges-Rovnyak spaces is given by the observations that follow.

Given a positive Borel measure $\mu$ on $[0,1)$, define a map $V_{\mu}$, called the normalized Cauchy transform, from $L^{1}(\mu)$ to the functions defined on $\mathbb{C} \backslash \mathbb{T}$ by

$$
V_{\mu} f(z):=\frac{\int_{0}^{1} \frac{f\left(e^{2 \pi i x}\right)}{1-z e^{-2 \pi i x}} d \mu(x)}{\int_{0}^{1} \frac{1}{1-z e^{-2 \pi i x}} d \mu(x)}
$$

Poltoratskiı proved in [6] that $V_{\mu}$ maps $L^{2}(\mu)$ to the de Branges-Rovnyak space $\mathcal{H}(b)$, where $b(z)$ is the inner function associated to $\mu$ via the Herglotz representation theorem. Poltoratskir also proved that $V_{\mu}$ is the inverse of a unitary operator that is a rank one perturbation of the unilateral shift as given by Clark [16], and hence $V_{\mu}$ is unitary.

Proposition 1. Assume the hypotheses of Theorem 1. Then, for $z \in \mathbb{D}$,

$$
V_{\mu} f(z)=\sum_{n=0}^{\infty}\left\langle f, g_{n}\right\rangle z^{n}
$$

Proof. Define

$$
F(z):=\int_{0}^{1} \frac{1}{1-z e^{-2 \pi i x}} d \mu(x) .
$$

That is, $F(z)$ is the Cauchy integral of $\mu$, which is analytic on $\mathbb{D}$. It is easily seen that

$$
F(z)=\sum_{n=0}^{\infty} \widehat{\mu}(n) z^{n}
$$

By Label (9), $\operatorname{Re}(F(z))>1 / 2$ for $z \in \mathbb{D}$, and, hence, $1 / F(z)$ is also analytic on $\mathbb{D}$. Writing $1 / F(z)=\sum_{n=0}^{\infty} c_{n} z^{n}$, we have $1=\sum_{n=0}^{\infty}\left(\sum_{k=0}^{n} c_{k} \widehat{\mu}(n-k)\right) z^{n}$, and so $\sum_{k=0}^{n} c_{k} \widehat{\mu}(n-k)=0$ for all $n \geq 1$. Then, using Label (3), an inductive argument shows that $g_{n}=\sum_{i=0}^{n} \overline{c_{n-i}} e_{i}$ for all $n$. The $c_{n}$ are unique by Gaussian elimination, so, in fact, $c_{n}=\alpha_{n}$ for all $n$, the $\alpha_{n}$ as in Lemma 2 . Hence,

$$
\frac{1}{F(z)}=\sum_{n=0}^{\infty} \alpha_{n} z^{n}
$$

It is also clear that

$$
\int_{0}^{1} \frac{f\left(e^{2 \pi i x}\right)}{1-z e^{-2 \pi i x}} d \mu(x)=\sum_{n=0}^{\infty}\left\langle f, e_{n}\right\rangle z^{n}
$$

Therefore, we have

$$
\begin{aligned}
\frac{\int_{0}^{1} \frac{f\left(e^{2 \pi i x}\right)}{1-z e^{-2 \pi i x}} d \mu(x)}{\int_{0}^{1} \frac{1}{1-z e^{-2 \pi i x}} d \mu(x)} & =\left(\sum_{n=0}^{\infty}\left\langle f, e_{n}\right\rangle z^{n}\right)\left(\sum_{m=0}^{\infty} \alpha_{m} z^{m}\right) \\
& =\sum_{n=0}^{\infty}\left(\sum_{i=0}^{n}\left\langle f, \overline{\alpha_{n-i}} e_{i}\right\rangle\right) z^{n} \\
& =\sum_{n=0}^{\infty}\left\langle f, g_{n}\right\rangle z^{n} .
\end{aligned}
$$

Two of the main results in [6] are Theorems 2.5 and 2.7, which together show that the Fourier series of $V_{\mu} f(z)$ converges to $f$ in the $L^{2}(\mu)$ norm provided that $\mu$ is singular. Combining this together 
with Proposition 1 recovers our Theorem 1 . Adding Clark's result that implies that $V_{\mu}$ is unitary, we recover the Plancherel identity.

Poltoratskir's results are more general than our Theorem 1 in the following way: if $\mu$ has an absolutely continuous component and a singular component, then for any $f \in L^{2}(\mu)$, the Fourier series of $V_{\mu} f$ converges to $f$ in norm with respect to the singular component. The Fourier series cannot in general converge to $f$ with respect to the absolutely continuous component of $\mu$ since the nonnegative exponentials are incomplete. It is unclear whether for such a $\mu$ every $f$ can be expressed in terms of a bi-infinite Fourier series. For singular $\mu$, our Theorem 1 guarantees norm convergence of the Fourier series of $V_{\mu} f$ to $f$ as do Poltoratskiir's results. However, Poltoratskir also comments in [6] that the Fourier series converges pointwise $\mu$-a.e. to $f$.

\section{A Shannon Sampling Formula}

In [17], Strichartz introduces a sampling formula for functions that are bandlimited in a generalized sense. He considers functions whose spectra are contained in a certain compact set $K$ that is the support of a spectral measure $\mu$. If $F$ is a strongly $K$-bandlimited function, then he shows that it has an expression

$$
F(x)=\sum_{\lambda \in \Lambda} F(\lambda) \widehat{\mu}(x-\lambda)
$$

where $\Lambda$ is a spectrum for $L^{2}(\mu)$.

We will now prove a similar sampling formula for analogously bandlimited functions. Our formula does not rely on an exponential basis and hence holds even for non-spectral singular measures. (Indeed, it even holds for singular measures devoid of exponential frames.) The price paid for not using an exponential sequence dual to itself is that the samples $F(\lambda)$ are replaced by the less tidy $\sum_{j=0}^{n} \alpha_{n-j} F(j)$.

Theorem 5. Let $\mu$ be a singular Borel probability measure on $[0,1)$. Let $\left\{\alpha_{i}\right\}_{i=0}^{\infty}$ be the sequence of scalars induced by $\mu$ by Lemma 2. Suppose $F: \mathbb{R} \rightarrow \mathbb{C}$ is of the form

$$
F(y)=\int_{0}^{1} f(x) e^{-2 \pi i y x} d \mu(x)
$$

for some $f \in L^{2}(\mu)$. Then,

$$
F(y)=\sum_{n=0}^{\infty}\left(\sum_{j=0}^{n} \alpha_{n-j} F(j)\right) \widehat{\mu}(y-n),
$$

where the series converges uniformly in $y$.

Proof. By Theorem 1, $f$ may be expressed $f=\sum_{n=0}^{\infty} c_{n} e_{n}$, the convergence occurring in the $L^{2}(\mu)$ norm. We compute:

$$
\begin{aligned}
F(y) & =\int_{0}^{1} f(x) e^{-2 \pi i y x} d \mu(x) \\
& =\left\langle f, e_{y}\right\rangle \\
& =\left\langle\sum_{n=0}^{\infty} c_{n} e_{n}, e_{y}\right\rangle \\
& =\sum_{n=0}^{\infty} c_{n}\left\langle e_{n}, e_{y}\right\rangle \\
& =\sum_{n=0}^{\infty} c_{n} \widehat{\mu}(y-n) .
\end{aligned}
$$


Recall from Corollary 1 that

$$
c_{n}=\sum_{j=0}^{n} \alpha_{n-j} \widehat{f}(j)=\sum_{j=0}^{n} \alpha_{n-j} F(j)
$$

where the $\alpha_{n}$ are defined by Lemma 2. Combining these computations, we obtain that for any $y \in \mathbb{R}$,

$$
F(y)=\sum_{n=0}^{\infty}\left(\sum_{j=0}^{n} \alpha_{n-j} F(j)\right) \widehat{\mu}(y-n) .
$$

Let $S_{k}:=\sum_{n=0}^{k} c_{n} e_{n}$. Since $S_{k} \rightarrow f$ in the $L^{2}(\mu)$ norm and the Fourier transform $\mathcal{F}: L^{2}(\mu) \rightarrow$ $L^{\infty}(\mathbb{R})$ is bounded, $\left\{\mathcal{F} S_{k}\right\} \rightarrow \mathcal{F} f$ in $L^{\infty}(\mathbb{R})$. Then, because $\mathcal{F} S_{k}(y)=\sum_{n=0}^{k} c_{n} \widehat{\mu}(y-n)$, we have that $\sum_{n=0}^{\infty} c_{n} \widehat{\mu}(y-n)$ and hence Label (10) converges uniformly in $y$ to $\mathcal{F} f(y)$.

It should be noted that, in contradistinction to the sampling formula of Strichartz, the convergence of the series in Equation (10) does not follow from the Cauchy-Schwarz inequality because it is possible that $\sum_{n=0}^{\infty}|\widehat{\mu}(y-n)|^{2}=+\infty$.

Author Contributions: The authors contributed equally to this work.

Conflicts of Interest: The authors declare no conflict of interest.

\section{References}

1. Jorgensen, P.E.T.; Pedersen, S. Dense analytic subspaces in fractal $L^{2}$-spaces. J. Anal. Math. 1998, 75, 185-228.

2. Duffin, R.J.; Schaeffer, A.C. A class of nonharmonic Fourier series. Trans. Amer. Math. Soc. 1952, 72, 341-366.

3. Lai, C.K. Spectral Analysis on Fractal Measures and Tiles. Ph.D. Dissertation, The Chinese University of Hong Kong, Hong Kong, China, 2012.

4. Dutkay, D.E.; Lai, C.K. Uniformity of measures with Fourier frames. Adv. Math. 2014, 252, 684-707.

5. Kwapień, S.; Mycielski, J. On the Kaczmarz algorithm of approximation in infinite-dimensional spaces. Studia Math. 2001, 148, 75-86.

6. Poltoratskiı̌, A.G. Boundary behavior of pseudocontinuable functions. Algebra i Analiz 1993, 5, 189-210. English translation in St. Petersb. Math. 1994, 5, 389-406.

7. Kaczmarz, S. Angenäherte Auflösung von Systemen linearer Gleichungen. Bulletin International de l'Académie Plonaise des Sciences et des Lettres 1937, 35, 355-357.

8. Kwapień, S.A.; Mycielski, J. Erratum to the paper: "On the Kaczmarz algorithm of approximation in infinite-dimensional spaces" [Studia Math. 148 (2001), no. 1, 75-86; MR1881441]. Studia Math. 2006, 176, 93.

9. Haller, R.; Szwarc, R. Kaczmarz algorithm in Hilbert space. Studia Math. 2005, 169, 123-132.

10. Li, S.; Ogawa, H. Pseudo-duals of frames with applications. Appl. Comput. Harmon. Anal. 2001, 11, 289-304.

11. Riesz, F.; Riesz, M. Über die Randwerte einer analytischen Funktion. Quatrième Congrès des Mathématiciens Scandinaves 1916, 27-44.

12. Koosis, P. Introduction to $H_{p}$ Spaces, 2nd ed.; Cambridge Tracts in Mathematics; Cambridge University Press: Cambridge, UK, 1998; Volume 115.

13. Herr, J.E. Fourier Series for Singular Measures and the Kaczmarz Algorithm. Ph.D. Thesis, Iowa State University, Ames, IA, USA, 2016.

14. Dutkay, D.E.; Han, D.; Weber, E. Continuous and discrete Fourier frames for fractal measures. Trans. Am. Math. Soc. 2014, 366, 1213-1235.

15. Dutkay, D.E.; Han, D.; Sun, Q.; Weber, E. On the Beurling dimension of exponential frames. Adv. Math. 2011, $226,285-297$. 
16. Clark, D.N. One dimensional perturbations of restricted shifts. J. Anal. Math. 1972, 25, 169-191.

17. Strichartz, R.S. Mock Fourier series and transforms associated with certain Cantor measures. J. Anal. Math. 2000, 81, 209-238.

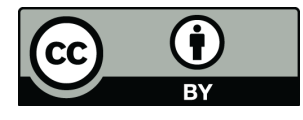

(C) 2017 by the authors; licensee MDPI, Basel, Switzerland. This article is an open access article distributed under the terms and conditions of the Creative Commons Attribution (CC BY) license (http://creativecommons.org/licenses/by/4.0/). 